\title{
Modulation of brain response to emotional conflict as a function of current mood in bipolar disorder: Preliminary findings from a follow-up state-based fMRI study
}

\author{
Gwladys Rey $^{\mathrm{a}, *}$, Martin Desseilles ${ }^{\mathrm{b}, \mathrm{c}}$, Sophie Favre ${ }^{\mathrm{b}}$, Alexandre Dayer ${ }^{\mathrm{b}}$, Camille Piguet ${ }^{\mathrm{a}}$, \\ Jean-Michel Aubry ${ }^{\mathrm{b}}$, Patrik Vuilleumier ${ }^{\mathrm{a}, \mathrm{d}}$ \\ a Laboratory for Behavioral Neurology and Imaging of Cognition, Department of Neuroscience, University of Geneva, 1 rue Michel Servet, 1211 Geneva, \\ Switzerland \\ ${ }^{\mathrm{b}}$ Department of Mental Health and Psychiatry, Division of Psychiatric Specialties, Mood Disorder Program, Geneva University Hospitals, Geneva, Switzerland \\ c Cyclotron Research Center, University of Liège, Liège, Belgium \\ d Department of Neurology, Geneva University Hospitals, Geneva, Switzerland
}

\section{A R T I C L E I N F O}

\section{Article history:}

Received 18 November 2013

Received in revised form

21 March 2014

Accepted 28 April 2014

\section{Keywords:}

Follow-up

Functional magnetic resonance imaging

(fMRI)

Interference

Euthymia

Hypomania

Depression

\begin{abstract}
A B S T R A C T
We used functional magnetic resonance imaging (fMRI) to examine affective control longitudinally in a group of patients with bipolar disorder (BD). Participants comprised 12 BD patients who underwent repeated fMRI scans in euthymic $(n=11)$, depressed $(n=9)$, or hypomanic $(n=9)$ states, and were compared with 12 age-matched healthy controls. During fMRI, participants performed an emotional face-word interference task with either low or high attentional demands. Relative to healthy controls, patients showed decreased activation of the cognitive control network normally associated with conflict processing, more severely during hypomania than during depression, but regardless of level of task demand in both cases. During euthymia, a decreased response to conflict was observed only during the high load condition. Additionally, unlike healthy participants, patients exhibited deactivation in several key areas in response to emotion-conflict trials - including the rostral anterior cingulate cortex during euthymia, the hippocampus during depression, and the posterior cingulate cortex during hypomania. Our results indicate that the ability of BD patients to recruit control networks when processing affective conflict, and the abnormal suppression of activity in distinct components of the default mode network, may depend on their current clinical state and attentional demand.
\end{abstract}

(c) 2014 Elsevier Ireland Ltd. All rights reserved.

\section{Introduction}

Bipolar disorder (BD) is a common and severe psychiatric illness characterized by episodes of extreme changes in mood state, ranging from major depression to mania. A better understanding of the cerebral mechanisms and impact of mood switching in $\mathrm{BD}$ is crucial in order to develop or improve treatments for this disorder. Functional neuroimaging studies in patients with BD have evidenced changes in limbic and prefrontal networks relative to healthy people (for reviews, see Blond et al., 2012; Townsend and Altshuler, 2012), suggesting anomalies in neural circuits involved in affective regulation that are potentially responsible for emotional homeostasis or its loss (Strakowski et al., 2012). However, few studies have sought to determine a neural signature

\footnotetext{
*Corresponding author. Tel.: +41 22379 5324; fax: +4122379 5402.

E-mail address: gwladys.rey@unige.ch (G. Rey).
}

of BD in different mood states. Furthermore, most of these previous imaging studies used cross-sectional designs in different patient groups, an approach that limits comparison between the different clinical states due to inter-subject differences. A few follow-up studies, however, scanned a single group of BD patients in two sessions - i.e., two mood states - allowing investigation of both mood-dependent and -independent anomalies (Caligiuri et al., 2006; Marchand et al., 2007; Kaladjian et al., 2009; Chen et al., 2010; Cerullo et al., 2012). These studies indicated that changes in the activity and connectivity of limbic and subcortical structures such as the basal ganglia, amygdala and hippocampus, as well as in the medial frontal cortices, may occur when switching between affective episodes to euthymia or vice-versa.

Here we aimed at investigating neural changes in affective control networks by scanning the same patients in three different mood states (euthymic, depressed, and hypomanic) while they performed an emotional interference control task. In this task, verbal affective labels were superimposed on fearful and joyful faces, producing either 
congruent or incongruent emotion conditions. In addition, we manipulated the cognitive demand across two task conditions (easy and hard) to examine whether attentional resource availability can modify the effect of emotion interference (Pourtois et al., 2013). We expected that this paradigm would modulate lateral prefrontal and anterior cingulate networks which are reliably modulated by emotional as well as non-emotional conflict conditions (Roberts and Hall, 2008; Chechko et al., 2012).

In previous studies of conflict processing in bipolar disorder, euthymic patients demonstrated decreased activation in frontalparietal networks and basal ganglia structures when processing non-emotional conflict (Kronhaus et al., 2006; Pompei et al., 2011) and task-irrelevant emotional information (Malhi et al., 2005). In addition, abnormal connectivity between prefrontal and limbic/ paralimbic areas has been reported in euthymic patients during a Stroop task (Pompei et al., 2011b). Only one neuroimaging study tested euthymic as well as depressed and manic BD patients while performing a Stroop task (Blumberg et al., 2003), finding both common and differential effects of conflict in distinct ventral prefrontal areas. On the other hand, results from working memory and sustained attention paradigms suggest that patients with BD may show insufficient modulation of lateral as well as ventromedial prefrontal areas in high-demanding conditions (e.g., Strakowski et al., 2004; Townsend et al., 2010; Pomarol-Clotet et al., 2012; Fernández-Corcuera et al., 2013). Thus, in patients with $\mathrm{BD}$, the processing of emotion-conflict trials in prefrontal networks might be differentially affected by the current mood state, either directly or indirectly through changes in attentional task demands.

By using our follow-up protocol in the same group of BD patients, we could directly assess whether an alteration of the control network response to conflict and task demand, with corresponding changes in prefrontal limbic areas, would be associated with changes in the mood state and vary according to the polarity of mood episodes (depression vs. hypomania). Based on previous findings, we expected that, during euthymia, patients with BD would show attenuated response in control networks during emotion-conflict trials, relative to healthy individuals, while depression and hypomania might lead to both common and mood-specific changes in the same networks. We also expected abnormal modulation of ventromedial and lateral prefrontal cortices by task load, although these effects were less predictable due to the novelty of the paradigm in patients with BD. More critically, based on previous follow-up studies of BD patients (e.g., Cerullo et al., 2012), we expected that activation patterns may change depending on mood episode polarity, specifically in limbic and prefrontal areas associated with emotion regulation and cognitive control.

\section{Methods}

2.1. Participants

The study protocol and the informed consent procedure received approval from the ethics committee of the Geneva University Hospitals. Table 1 provides a demographic and clinical description of the patients and controls.

Twelve subjects with BD were recruited from the outpatient unit "Mood Disorder Program" of the Geneva University Hospitals. The diagnosis of bipolar disorder was based on the DSM-IV-TR criteria and confirmed by the MiniInternational Neuropsychiatric Interview (M.I.N.I.; Sheehan et al., 1997) administered by a trained clinician. Eight patients met criteria for at least one other lifetime Axis I psychiatric disorder. All patients were medicated during the follow-up study. For seven patients, the medication changed qualitatively during the follow-up due to clinical considerations of the therapist in charge.

Twelve healthy controls matched for gender, age, handedness, and level of education were recruited via advertisements on web sites. These control participants had no history of neurological illness or Axis I psychiatric disorders as assessed by the M.I.N.I.

\subsection{Follow-up sessions}

During the follow-up period ( $14+3$ months in average), patients completed several experimental sessions, with an average interval of 2.8 months $( \pm 1.8)$

Table 1

Demographic and clinical variables.

\begin{tabular}{|c|c|c|c|c|c|}
\hline & \multicolumn{3}{|c|}{ Bipolar disorder patients $n=12$} & Healthy controls $n=12$ & Patients versus controls \\
\hline Age, years & \multicolumn{3}{|l|}{$42.6(11.4)$} & $41.3(12)$ & $t(22)=0.27, \mathrm{~ns}$ \\
\hline Gender & \multicolumn{3}{|l|}{$8 \mathrm{~m}, 4 \mathrm{f}$} & $8 \mathrm{~m}, 4 \mathrm{f}$ & \\
\hline Handedness & \multicolumn{3}{|c|}{3 left-handed, 1 ambidextrous } & 3 left-handed & \\
\hline Education, years & \multicolumn{3}{|l|}{$11.8(3.4)$} & $10.8(2.4)$ & $t(22)=0.79, \mathrm{~ns}$ \\
\hline Diagnosis & \multicolumn{3}{|c|}{7 BD-I; 4 BD-II; 1 BD-NS } & - & \\
\hline Age onset & \multicolumn{3}{|c|}{$20.8(8.9)$} & - & \\
\hline Illness duration & \multicolumn{3}{|l|}{$21.8(9.9)$} & - & \\
\hline \multicolumn{6}{|l|}{ Lifetime presence of: } \\
\hline $\mathrm{GAD}$ & \multicolumn{3}{|l|}{4} & - & \\
\hline Social phobia & \multicolumn{3}{|l|}{2} & - & \\
\hline Panic disorder & \multicolumn{3}{|l|}{1} & - & \\
\hline $\mathrm{OCD}$ & \multicolumn{3}{|l|}{1} & - & \\
\hline PTSD & \multicolumn{3}{|l|}{1} & - & \\
\hline ADHD & \multicolumn{3}{|l|}{3} & - & \\
\hline Subst. use disorder & \multicolumn{3}{|c|}{4 (2 past, 2 current) } & - & \\
\hline \multicolumn{6}{|l|}{ Medication: } \\
\hline Mood stabilizers & \multicolumn{3}{|c|}{ 9-12 (lithium, lamotrigine, valproate) } & - & \\
\hline Antipsychotics & \multicolumn{3}{|c|}{$6-8$} & - & \\
\hline Antidepressants & & - & \\
\hline Benzodiazepines & & - & \\
\hline Psychostimulants & \multicolumn{3}{|c|}{2 (for comorbid ADHD) } & - & \\
\hline Mood state & Euthymic (E) & Depressed (D) & Hypomanic (H) & Controls (C) & Group/mood comparison \\
\hline Individuals, $n$ & 11 & 9 & 9 & 12 & \\
\hline Sessions, $n$ & 17 & 13 & 12 & 24 & \\
\hline YMRS score & $1.9(2.0)$ & $0.5(0.6)$ & $13.3(3.3)$ & $0.5(1.1)$ & $H>E=D=C$ \\
\hline MADRS-S score & $4.3(3.2)$ & $15.3(3.4)$ & $1.6(1.6)$ & $1.0(0.9)$ & $D>E>H=C$ \\
\hline
\end{tabular}

Mean (standard deviation) are provided for age, illness duration and clinical scores. Abbreviations: $G A D=$ general anxiety disorder; OCD $=$ obsessive-compulsive disorder; PTSD $=$ post-traumatic stress disorder; $\mathrm{ADHD}=$ attention deficit and hyperactivity disorder (reported in the patients' medical records). 
between successive sessions. Each session began with a systematic psychometric assessment of mood including the clinician-rated Young Mania Rating Scale (YMRS; Young et al., 1978; French version: Favre et al., 2003), the self-rated MontgomeryÅsberg Depression Rating Scale (MADRS-S; Svanborg and Asberg, 1994; French version: Bondolfi et al., 2010), and the self-rated Internal State Scale (Bauer et al., 1991). Based on these scales, we classified the affective status of the patients in each session in three different mood states labeled as hypomania/euthymia/ depression. For the YMRS score, we used the standard cut-off (6) of the French version (Favre et al., 2003); for the MADRS-S, we used a cut-off of 12 (e.g. Hedborg and Muhr, 2011). In addition, ISS scores (Bauer et al., 2000) were used to categorize sessions associated with MADRS-S scores between 10 and 12 (two cases). These criteria yielded three categories of "hypomania" (YMRS score $\geq 6$; MADRS-S score $<10$ ), "depression" (MADRS-S score $\geq 12$, or 10-12 with ISS categorization into depression; YMRS <6), and "euthymia" (MADRS-S score $<10$; YMRS score $<6$ ). Applying these criteria to our sample, we obtained data from six patients in the three mood states, from five patients in two states, and from one patient in one state only. For each patient, one or two sessions were obtained in the same mood state (see Table 1 for distribution of the 42 sessions).

To control for any effect of habituation or learning, we also scanned healthy control participants in two sessions separated by an average of 2.1 months $( \pm 0.4)$.

\subsection{Emotional conflict task}

We developed this task based on the emotional conflict task used by Etkin et al. (2006). Fearful and joyful faces (half female, half male) were presented with the French words "joie" [joy] or "peur" [fear] written across them. Subjects were asked to report the emotional expression of the face, by pressing one of two response buttons, while ignoring the word. We also modulated the attentional demand across two conditions. In the low load (easiest) condition, each emotion category (joy or fear) was always associated with the same response button. In the high load (most difficult) condition, the response button depended on the gender (Fig. 1). Task implementation resulted in a 2 (Load) $\times 2$ (Congruence) $\times 2$ (Emotion) design with 24 trials for each of the eight conditions (see Supplementary material for further details). During each fMRI session, participants performed a different version of the interference task (with a different response mapping), for which they were trained just before entering the scanner.

\subsection{Data acquisition and processing}

Neuroimaging data were collected using a 3T Magnetom TIM Trio scanner (Siemens, Germany) and a 32-channel head coil (see Supplementary material for acquisition parameters).

Image processing and statistical analyses were carried out using standard procedures implemented in SPM8 (www.fil.ion.ucl.ac.uk/spm). Functional images were realigned, slice-time corrected, normalized to the standard Montreal Neurological Institute EPI template, and spatially smoothed with an 8-mm kernel.

\subsection{Data analysis}

\subsubsection{Clinical and behavioral analysis}

Clinical scores were compared with the Student $t$-test or the Mann-Whitney $U$-test. Accuracy (percentage of correct responses) and response times were analyzed for each group and each mood state via analyses of variance (ANOVAs). We also used ANOVAs to examine potential effects of mood and differences between patients and controls. Post hoc tests on significant results were planned via Tukey's honestly significant difference test.
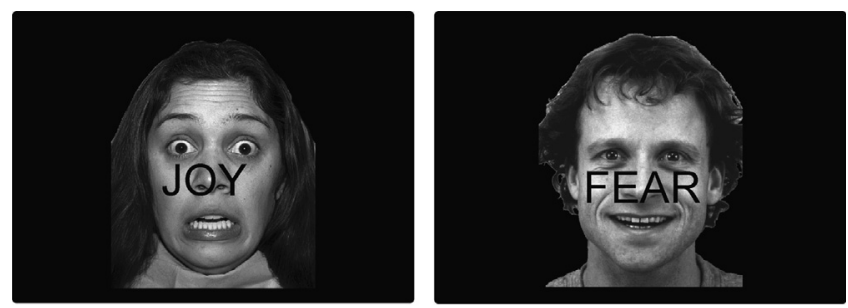

joyful face fearful face

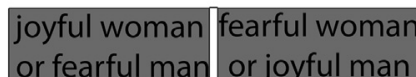

Fig. 1. Face-word interference task. Participants have to identify the facial expression while ignoring the word (low load task, left panel), or to identify both the facial expression and gender while ignoring the word (high load task, right panel).
2.5.2. Neuroimaging

Statistical inferences were drawn using a random effects General Linear Model (GLM) approach as implemented in SPM8. To avoid the possibility that some patients would differentially contribute to the second level analysis depending on the number of sessions in a given mood state at the first level, we constructed one single design matrix for each subject including all his sessions. For each session, we modeled eight conditions (using correct trials only): 2 Load (high, low) $\times 2$ Congruence (congruent, incongruent) $\times 2$ Emotion (joy, fear) as eight separate regressors convolved with the canonical form of the hemodynamic response function. We included six realignment parameters and one error condition as additional regressors of non-interest in the model, plus a high-pass filter of $128 \mathrm{~s}$ to account for low-frequency noise of the scanner. For each subject and each mood state, we computed one simple main effect contrast for each of the eight conditions separately (against implicit baseline), which was then used for within-group analyses. We also generated individual statistical images for the specific pairwise contrasts of [incongruent vs. congruent trials], [high load vs. low load trials], and for the interaction between load and congruence [high load (incongruent-congruent) vs. low load (incongruent-congruent)]. These were used for the subsequent between-group and between-state comparisons.

At the second level (random effect), between-group and between-mood analyses were performed using two-sample $t$-tests. For each of the pairwise contrasts described above, brain-activation patterns were compared between healthy controls $(\mathrm{HC})$ and patients in each mood state (HC vs. BD euthymia, HC vs. BD depression, HC vs. BD hypomania), and then in patients only between the three mood states (euthymia vs. depression vs. hypomania). Due to our first level analysis procedure, all patients in the second level analysis had the same number of contrast images in a given state. The resulting activation maps were thresholded at $p<0.001$ (uncorrected) with a minimum cluster size of 5 continuous voxels. Statistical inferences were corrected for multiple comparisons using Gaussian random field theory at the voxel level in a small spherical volume (radius $10 \mathrm{~mm}$ ) around a priori locations of structures of interest, taken from the relevant literature on conflict processing, attentional load, and bipolar disorder (see Table 2). Task conditions $\times$ group/mood interactions were further clarified and visualized by extracting parameter estimates of activity (beta weights) from the relevant clusters for each subject in each condition. When appropriate, these beta weights were submitted to additional ANOVAs to examine the interactions between experimental conditions and group or mood.

\section{Results}

\subsection{Clinical scores}

Mean scores (standard deviations) on clinical scales are provided for each group and each mood state in Table 1, together with statistical results from group and mood comparisons. These data confirmed significant mood differences between groups and between sessions in patients. Of note, treatment regimens did not differ between euthymia, depression, and hypomania (see contingency table in Supplementary Table S1).

\subsection{Behavioral responses}

Fig. 2 presents graphic plots of response times and accuracy. All conditions combined, patients were generally slower than controls, whatever the mood state $(p<0.01$ in euthymia and depression, $p<0.05$ in hypomania). Accuracy was comparable between groups and sessions, except for slightly lower performance during depression relative to euthymia $(p<0.05)$ and to healthy controls $(p<0.05)$.

Further analyses of behavioral data across the different task conditions confirmed a reliable Stroop interference effect (on both reaction times and accuracy) in controls as well as in patients in each mood state (see Supplementary material and Table S1). However, between-mood comparisons revealed a loss of the conflict effect on reaction times in patients during hypomania relative to euthymia (Mood $\times$ Congruence interaction, $p<0.05$ ), and between-group comparisons revealed a slightly greater effect of conflict on reaction times in patients during euthymia relative to controls (Group $\times$ Congruence interaction, $p<0.05)$. No other significant between-mood or betweengroup interaction was found. 
Table 2

Differential effect of conflict and load in patients compared to controls.

\begin{tabular}{|c|c|c|c|c|c|c|}
\hline Anatomical label & $\mathrm{BA}$ & MNI coord. & $Z$ & $p_{S V C}$ & Coord $_{\mathrm{SvC}}$ & $\operatorname{Ref}_{\text {SVC }}$ \\
\hline \multicolumn{7}{|c|}{ A. Patients during hypomania versus controls } \\
\hline \multicolumn{7}{|c|}{ HC vs. BD hypomania: Group $\times$ Congruence } \\
\hline \multicolumn{7}{|c|}{ Patients show deactivation to conflict [congruent > incongruent] } \\
\hline L dorsal PCC & 23 & $-9,-19,40$ & 3.32 & 0.021 & $-7,-20,45$ & $(5)$ \\
\hline $\mathrm{R}$ dorsal PCC & 23 & $3,-19,43$ & 3.30 & 0.023 & $-7,-20,45$ & (5) \\
\hline $\mathrm{L} / \mathrm{R}$ ventral PCC & 31 & $-3,-43,31$ & 3.36 & 0.019 & $-9,-44,37$ & (5) \\
\hline \multicolumn{7}{|c|}{ Patients show lower activation to conflict [incongruent $>$ congruent] than controls } \\
\hline L precG & 6 & $-48,8,40$ & 3.34 & 0.020 & $-43,5,35$ & $(1)$ \\
\hline L IFG & 45 & $-39,14,22$ & 3.86 & 0.004 & $-42,10,26$ & (3) \\
\hline R IFG & $9 / 46$ & $45,14,22$ & 4.25 & 0.001 & $48,10,31$ & (1) \\
\hline L orb. IFG/insula & 47 & $-39,23,-8$ & 3.63 & 0.009 & $-34,25,-1$ & (7) \\
\hline $\mathrm{R}$ orb. IFG & 47 & $45,35,-5$ & 4.17 & 0.002 & $36,31,-8$ & (9) \\
\hline L ant. MCC & 32 & $-3,14,37$ & 3.81 & 0.005 & $1,12,47$ & (1) \\
\hline $\mathrm{R}$ ant. MCC & 32 & $6,17,43$ & 3.89 & 0.004 & $1,12,47$ & (1) \\
\hline L pre-SMA & 6 & $9,14,46$ & 3.65 & 0.008 & $1,12,47$ & (1) \\
\hline R pre-SMA & 8 & $-3,14,55$ & 3.27 & 0.025 & $1,12,47$ & (1) \\
\hline L SPL & 7 & $-27,-64,52$ & 3.50 & 0.013 & $-22,-64,46$ & (2) \\
\hline R IPL/SPL & 40 & $42,-55,49$ & 3.34 & 0.020 & $41,-51,46$ & (1) \\
\hline L MTG/STG & $22 / 39$ & $-54,-55,13$ & 4.73 & $0.034^{*}$ & & \\
\hline R MTG/STG & $22 / 39$ & $51,-40,-11$ & 3.98 & 0.003 & $54,-47,-6$ & (5) \\
\hline L thalamus & - & $-12,-22,4$ & 4.08 & 0.002 & $-8,-27,5$ & (7) \\
\hline $\mathrm{R}$ thalamus & - & $18,-25,4$ & 4.04 & 0.002 & $16,-17,8$ & (8) \\
\hline L putamen & - & $-21,2,4$ & 3.53 & 0.012 & $-26,-4,4$ & (7) \\
\hline $\mathrm{R}$ putamen & - & $18,5,1$ & 3.46 & 0.014 & $24,4,2$ & (7) \\
\hline L pallidum & - & $-24,-10,4$ & 3.49 & 0.013 & $20,-8,-8$ & (7) \\
\hline \multicolumn{7}{|c|}{ HC vs. BD hypomania: Group $\times$ Load } \\
\hline \multicolumn{7}{|c|}{ Patients show lower activation to load [high $>$ low] than controls } \\
\hline $\mathrm{R}$ insula/IFG & 47 & $33,26,4$ & 4.31 & 0.001 & $34,25,3$ & (7) \\
\hline L IFG/MFG & 46 & $-39,29,19$ & 3.73 & 0.006 & $-48,30,16$ & (3) \\
\hline L precuneus & 7 & $-12,-67,40$ & 3.55 & 0.011 & $-18,-72,42$ & $(2)$ \\
\hline L IPL & 40 & $-36,-55,34$ & 3.30 & 0.022 & $-36,-49,34$ & $(6)$ \\
\hline $\mathrm{R}$ thalamus & - & $12,-10,1$ & 3.23 & 0.027 & $16,-17,8$ & $(8)$ \\
\hline \multicolumn{7}{|c|}{ B. Patients during depression versus controls } \\
\hline \multicolumn{7}{|c|}{ HC vs. BD depression: Group $\times$ Congruence } \\
\hline \multicolumn{7}{|c|}{ Patients show lower activation to conflict than controls [incongruent > congruent] } \\
\hline L precG & 6 & $-42,-4,52$ & 3.46 & 0.015 & $-38,-6,44$ & $(4)$ \\
\hline R IFG & 46 & $45,14,22$ & 3.39 & 0.018 & $48,10,31$ & (1) \\
\hline \multicolumn{7}{|c|}{ HC vs. BD depression: Group $\times$ Load $\times$ Congruence } \\
\hline \multicolumn{7}{|c|}{ Patients show deactivation to conflict [congruent $>$ incongruent] in high load condition, not controls } \\
\hline L hippocampus & - & $-27,-13,-14$ & 3.73 & 0.007 & $-24,-20,-10$ & (7) \\
\hline $\mathrm{R}$ hippocampus & - & $24,-10,-20$ & 3.40 & 0.018 & $24,-3,-17$ & (9) \\
\hline \multicolumn{7}{|c|}{ HC vs. BD depression: Group $\times$ Load } \\
\hline \multicolumn{7}{|c|}{ Patients show lower activation to load [high $>$ low] than controls } \\
\hline L/R thalamus & - & $-6,-7,4$ & 3.85 & 0.005 & $-14,-4,4$ & (6) \\
\hline \multicolumn{7}{|c|}{ C. Patients during euthymia versus controls } \\
\hline Anatomical label & BA & MNI Coord. & $\mathrm{Z}$ & $p_{S V C}$ & Coord $_{\text {svc }}$ & $\operatorname{Ref}_{\mathrm{SVC}}$ \\
\hline HC vs. BD euthymia: Group $\times C$ & & & & & & \\
\hline Patients show lower activatic & [incongrue & at] than controls & & & & \\
\hline R MFG & 9 & $39,20,34$ & 3.52 & 0.011 & $46,16,30$ & $(2)$ \\
\hline Patients show deactivation to & igruent $>$ in & not controls & & & & \\
\hline $\mathrm{L}$ rostral ACC & $32 / 10 / 24$ & $-12,38,4$ & 3.85 & 0.004 & $-18,41,-5$ & (7) \\
\hline L ventral PCC & $31 / 23$ & $-6,-40,28$ & 3.21 & 0.027 & $-9,-44,37$ & (5) \\
\hline HC vs. BD euthymia: Group $\times L$ & ience & & & & & \\
\hline Patients show lower activatic & [incongrue & t] than controls in & & & & \\
\hline${ }^{+}$Patients also show higher 0 & conflict [inc & ongruent] than con & & & & \\
\hline $\mathrm{R}$ pre-SMA/medial SFG ${ }^{+}$ & $6 / 8 / 32$ & $12,14,46$ & 4.05 & 0.002 & $10,18,38$ & (3) \\
\hline $\mathrm{L}$ re-SMA/medial SFG ${ }^{+}$ & $6 / 8 / 32$ & $-9,17,43$ & 3.49 & 0.014 & $-4,20,38$ & (3) \\
\hline R IFG/MFG & $9 / 46$ & $36,29,19$ & 3.40 & 0.018 & $32,38,22$ & (2) \\
\hline R MFG & 6 & $30,8,58$ & 4.34 & 0.001 & $28,1,57$ & (6) \\
\hline $\mathrm{L}_{\text {precG }}^{+}$ & $6 / 9$ & $-48,2,28$ & 3.52 & 0.008 & $-43,5,35$ & (1) \\
\hline R precG & 9 & $51,5,34$ & 3.28 & 0.026 & $48,10,31$ & (1) \\
\hline $\mathrm{L} \mathrm{IPL}^{+}$ & 40 & $-42,-49,40$ & 3.72 & 0.007 & $-36,-49,34$ & (6) \\
\hline R IPL & 40 & $36,-58,40$ & 3.59 & 0.010 & $38,-52,45$ & (6) \\
\hline
\end{tabular}

Note: All the regions reported are significantly activated at $p<0.001$ (uncorrected) and at $p<0.05$ after small volume correction (SVC) excepting for the following: $p_{S V C}: p$

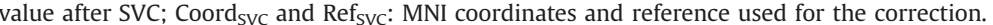

For small volume correction, we used findings from conflict processing literature: two meta-analyses of conflict processing studies: (1) Roberts and Hall (2008) and (2) Nee et al. (2007); two recent studies using face-word interference task (without load factor): emotional (3) Chechko et al. (2012) and non-emotional (4) Krebs et al. (2013); one study of task-induced deactivation during stroop performance: (5) Harrison et al. (2011). We also used (6) one meta-analysis from 221 studies with the factor 'Load' by Neurosynth.org (reverse inference, selection of the peak as a function of the Z score), and three meta-analyses of studies in BD patients: (7) Chen et al. (2011); (8) Houenou et al. (2011); (9) Delvecchio et al. (2012).

Abbreviations: ACC/MCC/PCC: anterior/middle/posterior cingulate cortex; IFG/MFG/SFG: inferior/medial/superior frontal gyrus; IPL/SPL: inferior/superior parietal lobe; precG: precentral gyrus; SMA: Supplementary Motor Area; MTG/STG: middle/superior temporal gyrus.

* significant activation at $p<0.05$ corrected over the entire brain volume (family-wise error correction). 


\section{3. fMRI results}

We first describe the effects found in controls and in patients in each mood state separately (voxelwise threshold of $p<0.001$ uncorrected), and then detail the between-group and betweenmood comparisons (voxelwise $p<0.001$, FWE $p<0.05$ with small volume correction).

\subsubsection{Brain activations in controls and in patients as a function of} mood state

A complete overview of brain activations in controls and in patients as a function of mood state is reported in Supplementary Tables S3-S6. Our main analysis focused on the emotional conflict

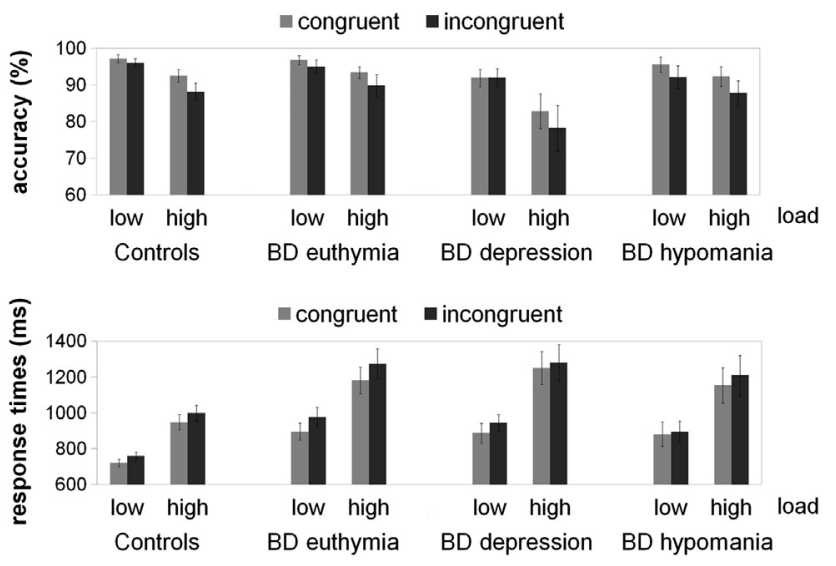

Fig. 2. Behavioral results. The mean $( \pm S E)$ response accuracy (top) and response times (bottom) are shown for healthy controls and bipolar disorder (BD) patients in each mood state. effect (different vs. same emotion conveyed by word and face, i.e., incongruent vs. congruent trials). Because the emotional category of faces was orthogonal to the task-relevant factors, fear- or joyspecific effects are not described in detail, but for completeness within-group results concerning Emotion category are also reported in the Supplementary Table S7.

3.3.1.1. Controls. In the critical contrast between incongruent versus congruent face-word trials, the healthy controls showed strong bilateral prefrontal activations predominating in the inferior frontal, middle frontal, and precentral gyri (IFG/MFG/precG), together with activations in the pre-Supplementary Motor Area (pre-SMA) and middle cingulate cortex (MCC), plus parietal, temporal, and thalamic regions (Fig. 3a and Supplementary Table S3). These effects are consistent with other studies on response conflict and executive control (e.g. Chechko et al., 2012). Partly overlapping regions also showed a significant main effect of task load (high $>$ low), including lateral prefrontal cortex (IFG/MFG/precG), pre-SMA, parietal cortex, thalamus, as well as insula (Supplementary Table S3).

3.3.1.2. Bipolar disorder. The patients showed only limited activations in the main contrast of incongruent versus congruent trials, and only during euthymia and depression (see Supplementary Tables S4 and S5). In both cases, we observed a main activation cluster in the left precG/IFG (Figs. 3b and c), but no recruitment of other prefrontal and parietal areas. In addition, during euthymia, we observed a significant Load $\times$ Congruence interaction in several regions (Fig. 3b). As indicated by further analyses of beta values extracted from these regions, euthymic patients showed significant activation to incongruent relative to congruent trials in the low load condition (but not in the high load condition) in the right precG/IFG, right inferior parietal lobe, and medial superior frontal gyrus (SFG).
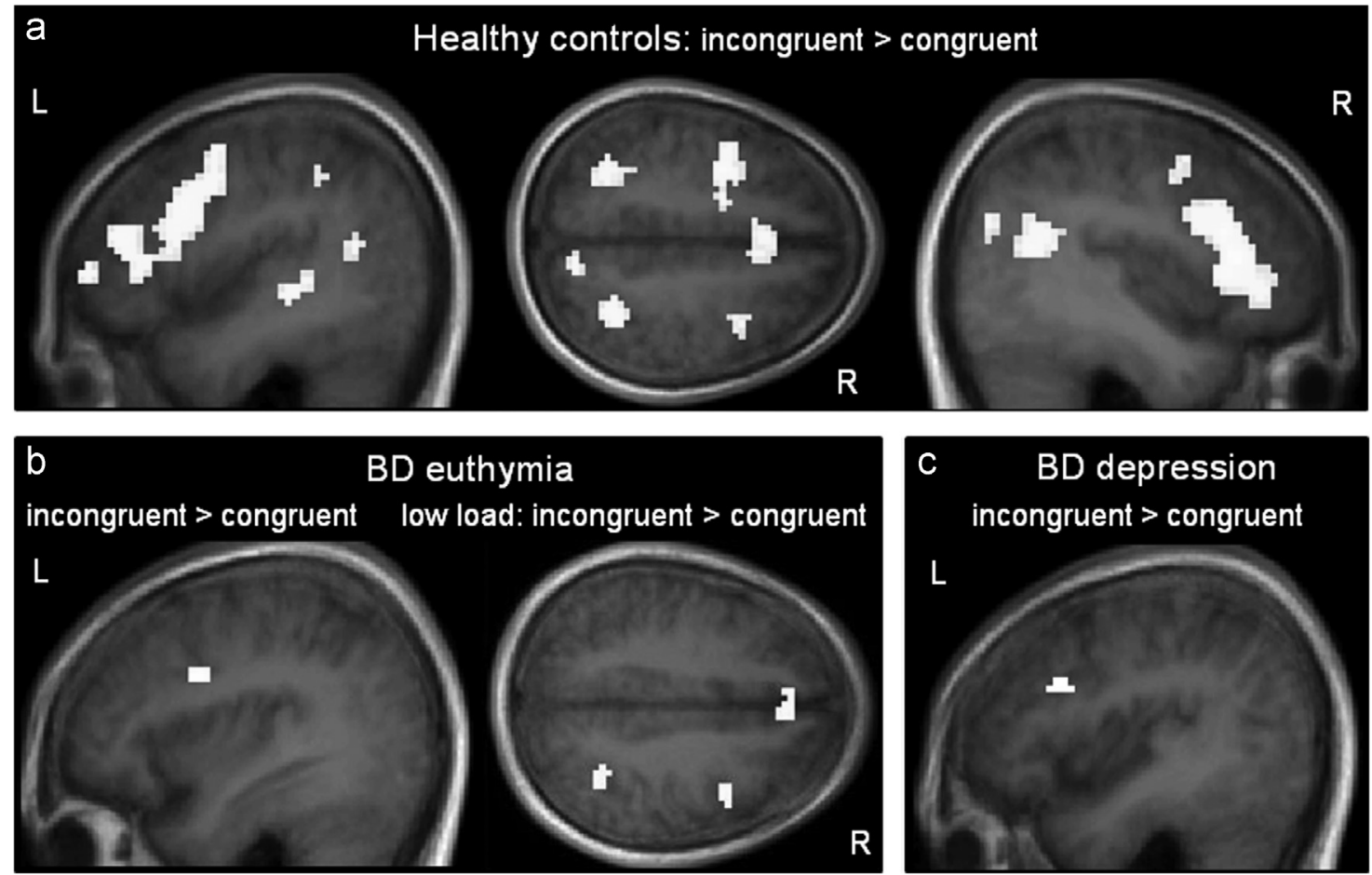

Fig. 3. Brain activations elicited by emotion conflict trials ( $p<0.001$ uncorrected). (a) In healthy controls: greater activation to incongruent than congruent trials was found in bilateral IFG/MFG/precG, plus pre-SMA/MCC, parietal cortex, and temporal regions. (b) In BD patients during euthymia: the same contrast (incongruent > congruent) activated only the left precG/IFG (left panel). Activations were also found in medial SFG, right precG and right angular gyrus for the same contrast (incongruent > congruent) only during the low load condition (right panel). Activations displayed here were obtained by inclusively masking the Load $\mathrm{x}$ Congruence interaction $(p<0.001$ unc.) with the contrast of "incongruent minus congruent trials" in the low load task ( $p<0.001$ unc.). (c) In BD patients during depression: the same contrast (incongruent $>$ congruent) activated only the left posterior IFG. L: left; R: right.

Please cite this article as: Rey, G., et al., Modulation of brain response to emotional conflict as a function of current mood in bipolar disorder: Preliminary findings from a.... Psychiatry Research: Neuroimaging (2014), http://dx.doi.org/10.1016/j.pscychresns.2014.04.016 
On the contrary, the patients still showed significant increases in lateral prefrontal areas, MCC/pre-SMA and parietal areas in the contrast high $>$ low load task, although this effect reached significance only during euthymia and depression (Supplementary Tables S4 and S5). Thus, while most prefrontal areas showed combined effects of congruence and load in healthy controls, only the effects of load were preserved in patients, at least during euthymia and depression. During hypomania, we found no increases for the incongruent compared with congruent trials, nor for the high compared with low load conditions (Supplementary Table S6).

Remarkably, unlike healthy controls, patients showed significant activations in the reverse contrast, i.e., congruent minus incongruent trials. Furthermore, these effects differed as a function of mood state. Thus, when euthymic, the patients showed deactivation to incongruent relative to congruent trials in the anterior cingulate cortex (ACC), implicating a large cluster covering both the pregenual and subgenual components of this area. Additional deactivations were also found in several other regions in the high load condition, including the MCC extending to the posterior cingulate cortex (PCC), and the postcentral gyrus (Supplementary Table S4). When hypomanic, the patients showed a different deactivation to incongruent trials (whatever the task load), mainly encompassing the dorsal PCC and MCC, but also the rostral ACC, as well as to a lesser degree the precuneus, postcentral gyrus, dorsolateral prefrontal cortex, and temporal areas (Supplementary Table S6). Finally, when depressed, patients showed a selective deactivation in response to incongruent trials in the left hippocampus, but only during the high load condition, leading to a significant Load $\times$ Congruence interaction (Supplementary Table S5).

\subsubsection{Between-group comparisons and effects of mood}

The above results were complemented by direct statistical comparisons between groups and states. Two-sample $t$-test contrasts between controls and patients in each mood state revealed significantly lower activation to conflict in BD patients in several prefrontal, parietal, and subcortical areas (Table 2). During hypomania, we found a significant reduction of activation in a large network implicated in cognitive control, i.e., precG/IFG, anterior MCC and preSMA, as well as parietal regions, thalamus, and putamen (Table 2A). During depression, lower activation to conflict for patients versus controls was found in the left precG and right IFG (Table 2B and Fig. 4). During euthymia, patients showed more limited reductions in activation. A significant decrease in responses to conflict was observed only in the right MFG, which also demonstrated a Group$\times$ Load $\times$ Congruence interaction in more detailed contrasts (see Table 2C). An additional analysis of beta values from this region indicated that patients were actually able to activate the right MFG

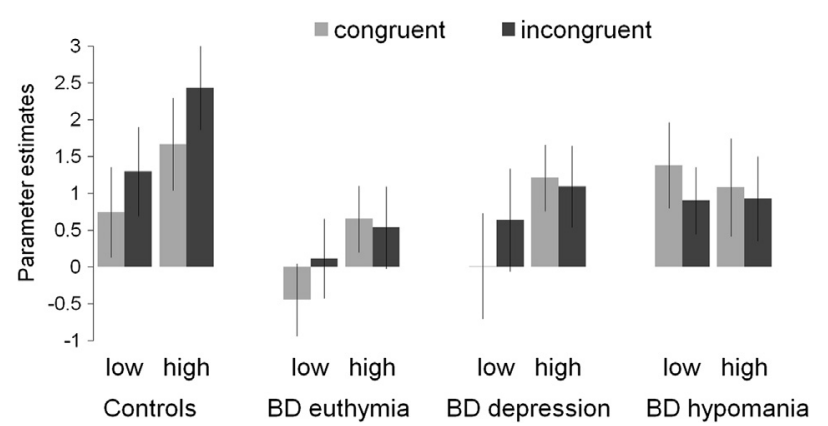

Fig. 4. Right IFG activity across conditions. Bar graphs show parameter estimates (mean, standard error) extracted from the right IFG cluster [peak at 45, 14, 22] that showed a significant Group-by-Congruence interaction in the comparison of patients during depression vs. controls, and also overlapped with the same interaction in the comparison of patients during hypomania vs. controls. for incongruent trials in low load (not high load) conditions, whereas healthy controls showed both an effect of conflict and an additional increase by high load in all conditions (see also Fig. 4). The same interaction was observed in other regions of the control network, including the pre-SMA/anterior MCC, bilateral prefrontal and parietal areas, where euthymic patients demonstrated an increase to conflict in low load conditions but controls exhibited a stronger effect of conflict in high load conditions.

In contrast to the differential effect of conflict, we found no difference between controls and patients during euthymia for the main effects of load (contrast high vs. low), and a difference limited to the thalamus during depression (Table 2B). Changes were larger during hypomania, however, with decreased activation in the IFG bilaterally, the parietal lobe and the thalamus (Table $2 \mathrm{~A}$ ) relative to the load effect of controls.

Our between-group analyses also confirmed the mood-specific deactivations to conflict in patients, which were absent in the controls. Specifically, relative to controls, patients showed a large conflict-induced deactivation in the rostral ACC during euthymia, plus a smaller deactivation in the ventral PCC (Table 2C and Fig. 5a). During hypomania, patients showed significantly greater deactivation in the PCC, both ventrally and dorsally (Table 2A and Fig. 5b). During depression, patients showed selective and bilateral deactivation in the hippocampus in the high load condition (see Group $\times$ Load $\times$ Congruence interaction in Table $2 \mathrm{~B}$ and Fig. $5 \mathrm{c}$ ).

Finally, two-sample $t$-tests were also used for direct comparisons of brain activity between the mood states within the patient group (Table 3). Compared with euthymia, hypomania was associated with decreased activation to conflict in the thalamus regardless of load, decreased activation to conflict in the preSMA/anterior MCC in the low load condition, and decreased activation to high load in lateral prefrontal and parietal areas. Compared with depression, euthymia was associated with deactivation to conflict trials in the rostral ACC. Of note, at a slightly more lenient threshold ( $p<0.005$ uncorrected), we also observed a deactivation to conflict in the rostral ACC during euthymia compared with hypomania (Mood $\times$ Congruence interaction), a deactivation to conflict in the dorsal PCC during hypomania compared with depression (Mood $\times$ Congruence interaction), and a deactivation to conflict in high load in the left hippocampus during depression compared with both euthymia and hypomania (Mood $\times$ Congruence $\times$ Load interactions). Thus, differential deactivation in the rostral ACC during euthymia, dorsal PCC during hypomania, and hippocampus during depression were generally confirmed in the direct comparisons between mood states, but with lower statistical values in some cases, probably due to our small population and lack of statistical power.

To summarize, patients with BD showed decreased activation to emotion-conflict trials in prefrontal control networks, particularly during depression and even more so during hypomania, with such changes being independent of task load. During euthymia, patients showed a weaker impairment, with preserved activation of the control network to conflict observed in the low load condition only (not high). In addition, unlike healthy controls, patients showed deactivations to emotion-conflict trials in specific midline brain regions that varied as a function of mood state.

For completeness, additional results for comparisons involving the Emotion factors are reported in Supplementary material (Table S7).

\subsection{Subsidiary analyses}

We examined potential changes in behavioral performance and BOLD activations due to habituation or learning across repeated sessions in controls. The session factor had no effect on behavioral measures and did not interact with the effect of Congruence on brain activity. It is therefore unlikely that habituation or learning 

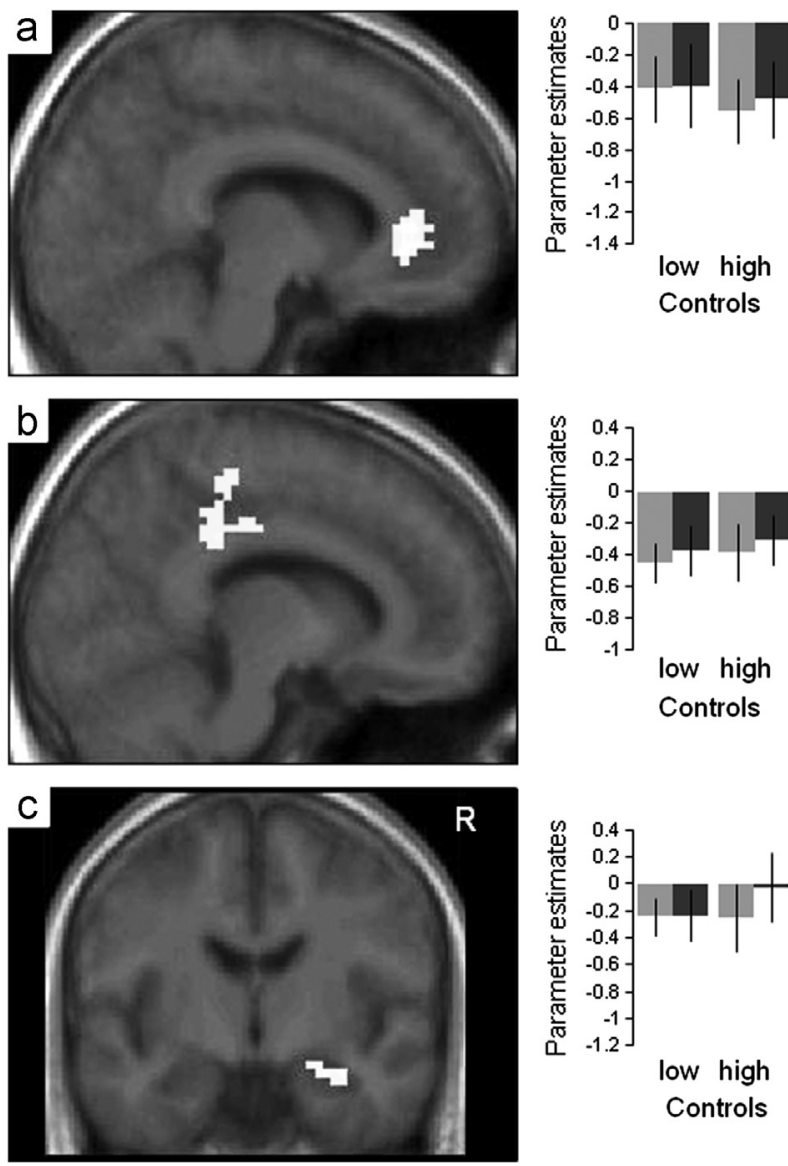
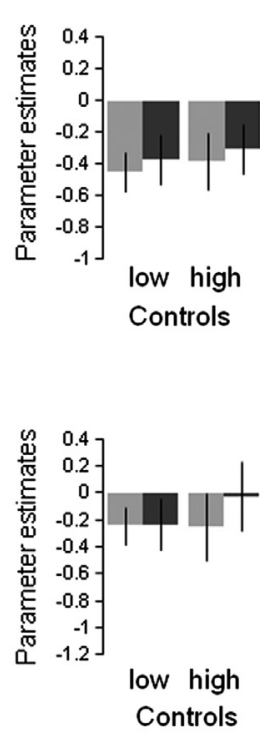

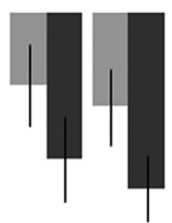

congruent

incongruent

low high

BD euthymia
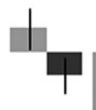

a congruent

$\square$ incongruent

low high

BD hypomania

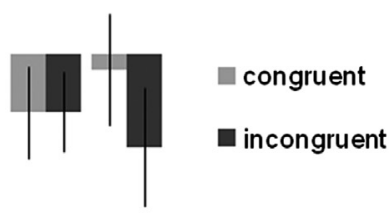

low high

BD depression

Fig. 5. Significant deactivation to conflict trials was observed in patients with BD compared to controls ( $p<0.001$ uncorrected). (a) During euthymia, patients deactivated the rostral ACC in response to incongruent compared to congruent trials, while controls did not (Group $\times$ Congruence). (b) During hypomania, patients deactivated the dorsal PCC in response to the same incongruent trials, while controls did not (Group $\times$ Congruence). c) Unlike controls, patients during depression deactivated the right hippocampus in response to incongruent trials but only in the high load condition (Group $\times$ Congruence $\times$ Load interaction). Bar graphs show the corresponding parameter estimates of activity (mean, SE) from these regions, plotted for the healthy controls and patients in the mood condition of interest. Additional data illustrating parameter estimates for all the three mood states in each region are provided in Supplementary Fig. S8. R: right.

affected the differences observed between the mood states in patients. Furthermore, due to individual differences in clinical course, session order did not correspond to the same mood states across patients.

\section{Discussion}

The present study used an emotional face-word interference task in conditions of low and high attentional demands to probe cognitive control networks in a group of BD patients who were prospectively followed across different mood states. In healthy controls we replicated prior findings of activation in a lateral prefrontal network in response to emotion conflict. In the same task conditions, patients showed a lower recruitment of this network, especially during mood disorder episodes. In addition, unlike controls, they demonstrated significant deactivations in distinct midline and limbic brain regions as a function of mood state.

In healthy controls, emotion conflict was associated with robust activation in a bilateral frontal-parietal network comprising the MCC and pre-SMA, a network typically active when subjects have to resolve interference (Nee et al., 2007; Roberts and Hall, 2008; Ovaysikia et al., 2011; Chechko et al., 2012). The same network also showed increased activation in high versus low load conditions, consistent with previous studies that manipulated task demand (Schwartz et al., 2005; Rottschy et al., 2012). In patients, on the other hand, we observed very limited recruitment of this control network on conflict trials. Direct comparison with controls confirmed a dramatic reduction of activation in patients, particularly during hypomania, which affected lateral prefrontal, medial superior frontal and parietal areas, as well as thalamus and putamen. Patients showed less impairment during depression, with reductions predominating in lateral prefrontal areas as compared with controls. During euthymia, the patients demonstrated reduced activation to conflict in the high load condition only, again affecting the medial and inferior lateral prefrontal areas.

Importantly, however, during both euthymia and depression, patients showed a relatively preserved ability to recruit the same network (bilateral prefrontal and parietal regions, pre-SMA/MCC) in response to higher task load, suggesting a specific impact of $\mathrm{BD}$ on the processing of emotion conflict rather than task difficulty per se or poor imaging signal. Furthermore, in these patients, the activation of cognitive control systems by conflict trials appeared to be limited by resource availability, hence possible during low load but exhausted in the high load task. The significant Congruence-by-Load interaction observed during euthymia accords with this interpretation, as it reflects a ceiling effect in the recruitment of this control network. On the other hand, during hypomania, no differential activation to high load was observed, and the comparison with controls as well as with euthymia indicated significant decreases bilaterally in the prefrontal areas. Thus, in the case of hypomania, the lack of activation to incongruence may reflect a more general disturbance that manifests itself beyond the emotion-conflict condition. 
Table 3

In patients, differential effect of conflict and load depending on mood state.

\begin{tabular}{|c|c|c|c|c|c|c|}
\hline Anatomical label & BA & MNI coord. & $Z$ & $p_{\text {SVC }}$ & Coord $_{\mathrm{svc}}$ & $\operatorname{Ref}_{\text {SVC }}$ \\
\hline \multicolumn{7}{|c|}{ BD depression vs. euthymia: Mood $\times$ Congruence } \\
\hline \multicolumn{7}{|c|}{ Deactivation to conflict [congruent > incongruent] during euthymia, not during depression } \\
\hline $\mathrm{L}$ rostral ACC & $32 / 10$ & $-12,41,-2$ & 3.51 & 0.013 & $-18,41,-5$ & (7) \\
\hline \multicolumn{7}{|c|}{ BD hypomania vs. euthymia: Mood $\times$ Congruence } \\
\hline \multicolumn{7}{|c|}{ Lower activation to conflict [incongruent > congruent] during hypomania than during euthymia } \\
\hline $\mathrm{R}$ thalamus & - & $18,-25,7$ & 3.58 & 0.010 & $16,-17,8$ & (8) \\
\hline \multicolumn{7}{|c|}{ BD hypomania vs. euthymia: Mood $\times$ Load } \\
\hline \multicolumn{7}{|c|}{ Lower activation to load [high $>$ low] during hypomania than during euthymia } \\
\hline R MFG/IFG & 46 & $39,35,19$ & 3.39 & 0.021 & $40,34,24$ & (6) \\
\hline L MFG & 10 & $-30,38,22$ & 3.26 & 0.029 & $-38,34,30$ & (6) \\
\hline L precG & 6 & $-45,-4,28$ & 3.91 & 0.004 & $-44,1,26$ & (6) \\
\hline L IPL & 40 & $-42,-55,37$ & 3.47 & 0.017 & $-36,-49,34$ & (6) \\
\hline L precuneus & 7 & $-12,-67,40$ & 3.24 & 0.030 & $-18,-72,42$ & (2) \\
\hline \multicolumn{7}{|c|}{ BD hypomania vs. euthymia: Mood $\times$ Load $\times$ Congruence } \\
\hline \multicolumn{7}{|c|}{ Activation to conflict [incongruent $>$ congruent] in low load during euthymia, not hypomania } \\
\hline L pre-SMA/ant. MCC & 32 & $-9,17,43$ & 3.88 & 0.005 & $-4,20,38$ & (3) \\
\hline \multicolumn{7}{|c|}{ Deactivation to conflict [congruent > incongruent] in high load during euthymia, in low load during hypomania } \\
\hline R medial SFG & $6 / 8$ & $9,8,64$ & 3.16 & 0.037 & $8,14,66$ & (3) \\
\hline R MFG & 8 & $39,17,43$ & 3.23 & 0.032 & $45,12,40$ & (1) \\
\hline R MCC & $24 / 32$ & $6,11,31$ & 3.70 & 0.008 & $10,18,38$ & (3) \\
\hline L MCC & 24 & $-12,2,37$ & 3.62 & 0.011 & $-6,4,40$ & $(2)$ \\
\hline \multicolumn{7}{|c|}{ BD depression vs. hypomania: Mood $\times$ Congruence } \\
\hline \multicolumn{7}{|c|}{ Activation to conflict [incongruent $>$ congruent] during depression, not hypomania } \\
\hline R MTG & 37 & $48,-40,-8$ & 3.66 & 0.009 & $54,-47,-6$ & $(5)$ \\
\hline \multicolumn{7}{|c|}{ BD depression vs. hypomania: Mood $\times$ Load } \\
\hline \multicolumn{7}{|c|}{ Activation to load [high $>$ low] during depression, not hypomania } \\
\hline R IFG/insula & 13 & $42,26,10$ & 3.61 & 0.010 & $42,23,1$ & $(8)$ \\
\hline
\end{tabular}

Note: For abbreviations and explanations, see Table 2.

To our knowledge, our study is the first to investigate brain function during an emotional picture-word interference task in BD patients and to do so across different mood states in the same individuals. However, our findings are consistent with previous Stroop studies that reported reduced activation to conflict trials in IFG/MFG, medial SFG/SMA, parietal cortex, and basal ganglia in euthymic BD patients (Malhi et al., 2005; Strakowski et al., 2005; Kronhaus et al., 2006; Roth et al., 2006; Pompei et al., 2011). Stroop-like conflict has rarely been studied during affective episodes, but decreased activity in the IFG, together with hypoactivation of the basal ganglia, thalamus and/or parietal areas, was reported during inhibition and emotion categorization tasks in manic and depressed patients, as well as euthymic patients (Mazzola-Pomietto et al., 2009; Foland-Ross et al., 2012; Townsend et al., 2012; Vizueta et al., 2012). Together with our findings, these studies suggest that functional anomalies in ventrolateral prefrontal-striato-pallido-thalamic networks (Strakowski et al., 2012) may persist in BD patients across clinical changes, but to a different degree depending on mood and available cognitive resources. This pattern provides important novel insights into the neural circuits affected by the disorder.

In addition to these functional changes in prefrontal control networks, our patients also demonstrated relative deactivations in response to emotion-conflict trials, which affected different areas depending on mood state. Such deactivations were never observed in controls. Most notably, when they were euthymic, the patients exhibited a selective deactivation of rostral ACC on the conflict trials. Interestingly, deactivation of ventromedial prefrontal areas and subgenual ACC has been previously reported in euthymic patients during a color-word Stroop task (Kronhaus et al., 2006). A central involvement of the rostral ACC in the pathophysiology of both unipolar and bipolar mood disorders has been documented (e.g. Holtzheimer et al., 2012; Emsell et al., 2014). The rostral ACC overlaps with the default-mode network (DMN), which is typically active during the resting state (Qin and Northoff, 2011), and its hyperactivity at rest may reflect excessive self-referential processing (Lemogne et al., 2012). Although we did not assess this dimension directly, one possible explanation for rostral ACC deactivation in our study may be that euthymic patients disengaged self-referential processes to a greater extent when they had to resist interference. Conversely, they appeared much less - or not at all - able to reduce rostral ACC activity during depression (see Supplementary Fig. S8).

Instead, during depression, patients exhibited a selective deactivation of the hippocampus in response to conflict trials, specifically when task demand was high. Previously implicated in BD (Chen et al., 2011; Vederine et al., 2011), the hippocampus and adjacent medial temporal regions are involved in episodic memory retrieval (Huijbers et al., 2011) and also part of the DMN (Buckner et al., 2008). Decreased activity in hippocampal regions in trials that require maximal cognitive control might reflect a reduction in internally generated thoughts and memories, which tend to be more prevalent and intrusive under less focused attentional conditions in depressive states (Hertel, 1998; Davis and NolenHoeksema, 2000; Levens et al., 2009).

Finally, during hypomania, patients exhibited a pattern of conflict-related deactivation consistent with a task-negative response of the DMN, involving midline structures in both the posterior (dorsal PCC/posterior MCC/precuneus) and anterior (rostral ACC and anterior medial prefrontal cortex) brain regions. Direct comparison with controls also confirmed a significant deactivation in the PCC, a region thought to signal environmental changes and the need for commensurate adaptation of behavioral strategy based on action outcome (Pearson et al., 2011). Thus, a decreased recruitment of the PCC during conflict processing might reflect the lack of adequate behavior adjustment during mania including insufficient monitoring of and adjustment to errors. Indeed, when hypomanic, our patients showed lower accuracy in 
incongruent trials but not slower responding (or less slowing than during euthymia), a performance pattern (speed at the expense of accuracy) consistent with manic impulsivity (Strakowski et al., 2010).

Taken together, decreased engagement of (especially prefrontal) cognitive control networks in response to emotion conflict and concurrent deactivation of specific components of the DMN depending on current mood state are consistent with abnormal dynamics of large-scale brain networks in BD (Chai et al., 2011; Pomarol-Clotet et al., 2012; Fernández-Corcuera et al., 2013) - a finding that has been more widely documented in unipolar depression disorder (Marchetti et al., 2012; Whitfield-Gabrieli and Ford, 2012). DMN and task-positive networks typically show reciprocal activations during rest versus task performance, respectively (Fox et al., 2005). Here we found that task-positive networks were poorly modulated by task-related factors in BD patients, possibly due to a "saturation" of cognitive resources and reduced ability to control emotion-related conflicts, whereas components of the DMN showed abnormally increased (and anatomically dissociable) deactivations in conflict conditions, which were not seen in healthy controls.

Though providing novel and robust findings, our study is not without limitations. These include the relatively modest sample size (though comparable with other follow-up studies in BD; e.g. Cerullo et al., 2012); the inherent clinical heterogeneity of BD and related comorbidities, especially the inclusion of different subtypes of $\mathrm{BD}$, as well as the mixing of left- and right-handed subjects; our use of a self-rating scale for evaluation of depression but a clinician-rated scale for mania; and the fact that we could not see all 12 patients in the three possible mood states. In addition, we could not control for potential effects of medication and its changes over the follow-up period. However, medication has been shown to diminish behavioral and neural differences between patient groups and controls (Hafeman et al., 2012), so that this confound is unlikely to explain our main findings. Further, and this is the major strength of our work, following such patients and obtaining adequate task performance in fMRI condition across several sessions and mood states is rather challenging, but the current study provides an important first attempt to do so.

Our findings provide new insights into potential pathophysiological processes underlying mood switching in BD, by better characterizing the functional anomalies of control network that are sensitive to specific task demands and modulated by mood state. Conflict-induced deactivations in the rostral anterior cingulate, hippocampus, and posterior cingulate might pinpoint neural substrates that constitute a specific functional signature of brain changes and mood conditions. In the future, in clinical practice, one can envision the use of such markers (or related brainactivation profiles) as additional supports for early detection of mood switches and adjustment of pharmacological or psychotherapeutic treatment.

\section{Acknowledgments}

This study was supported by Grants from the Swiss Academy of Medical Sciences and the Boninchi Foundation, and the Foremane Chair Fund from the Geneva Academic Society awarded to PV. We thank Sebastian Rieger and Bruno Bonet for their help with the neuroimaging set-up and data collection.

\section{Appendix A. Supplementary material}

Supplementary data associated with this article can be found in the online version at http://dx.doi.org/10.1016/j.pscychresns.2014.04.016.

\section{References}

Bauer, M.S., Crits-Christoph, P., Ball, W.A., Dewees, E., McAllister, T., Alahi, P., Cacciola, J., Whybrow, P.C., 1991. Independent assessment of manic and depressive symptoms by self-rating. Scale characteristics and implications for the study of mania. Arch. Gen. Psychiatry 48, 807-812.

Bauer, M.S., Vojta, C., Kinosian, B., Altshuler, L., Glick, H., 2000. The Internal State Scale: replication of its discriminating abilities in a multisite, public sector sample. Bipolar Disord. 2, 340-346.

Blond, B.N., Fredericks, C.A., Blumberg, H.P., 2012. Functional neuroanatomy of bipolar disorder: structure, function, and connectivity in an amygdala-anterior paralimbic neural system. Bipolar Disord. 14, 340-355.

Blumberg, H.P., Leung, H.-C., Skudlarski, P., Lacadie, C.M., Fredericks, C.A., Harris, B. C., Charney, D.S., Gore, J.C. Krystal, J.H., Peterson, B.S., 2003. A functional magnetic resonance imaging study of bipolar disorder: state- and trait-related dysfunction in ventral prefrontal cortices. Arch. Gen. Psychiatry 60, 601-609.

Bondolfi, G., Jermann, F., Rouget, B.W., Gex-Fabry, M., McQuillan, A., DupontWillemin, A., Aubry, J.-M., Nguyen, C., 2010. Self- and clinician-rated Montgomery-Asberg Depression Rating Scale: evaluation in clinical practice. J. Affect. Disord. 121, 268-272.

Buckner, R.L., Andrews-Hanna, J.R., Schacter, D.L., 2008. The brain's default network: anatomy, function, and relevance to disease. Ann. N. Y. Acad. Sci. 1124, $1-38$.

Caligiuri, M.P., Brown, G.G., Meloy, M.J., Eberson, S., Niculescu, A.B., Lohr, J.B., 2006. Striatopallidal regulation of affect in bipolar disorder. J. Affect. Disord. 91, 235-242.

Cerullo, M.A., Fleck, D.E., Eliassen, J.C. Smith, M.S., DelBello, M.P., Adler, C.M., Strakowski, S.M., 2012. A longitudinal functional connectivity analysis of the amygdala in bipolar I disorder across mood states. Bipolar Disord. 14, 175-184.

Chai, X.J., Whitfield-Gabrieli, S., Shinn, A.K., Gabrieli, J.D.E., Nieto Castañón, A., McCarthy, J.M., Cohen, B.M., Ongür, D., 2011. Abnormal medial prefrontal cortex resting-state connectivity in bipolar disorder and schizophrenia. Neuropsychopharmacology 36, 2009-2017.

Chechko, N., Kellermann, T., Zvyagintsev, M., Augustin, M., Schneider, F., Habel, U., 2012. Brain circuitries involved in semantic interference by demands of emotional and non-emotional distractors. PLoS One 7, e38155.

Chen, C.-H., Suckling, J., Lennox, B.R., Ooi, C., Bullmore, E.T., 2011. A quantitative meta-analysis of fMRI studies in bipolar disorder. Bipolar Disord. 13, 1-15.

Chen, C.-H., Suckling, J., Ooi, C., Jacob, R., Lupson, V., Bullmore, E.T., Lennox, B.R., 2010. A longitudinal fMRI study of the manic and euthymic states of bipolar disorder. Bipolar Disord. 12, 344-347.

Davis, R.N., Nolen-Hoeksema, S., 2000. Cognitive inflexibility among ruminators and nonruminators. Cognit. Ther. Res. 24, 699-711.

Delvecchio, G., Fossati, P., Boyer, P., Brambilla, P., Falkai, P., Gruber, O., Hietala, J. Lawrie, S.M., Martinot, J.-L., McIntosh, A.M., Meisenzahl, E., Frangou, S., 2012. Common and distinct neural correlates of emotional processing in Bipolar Disorder and Major Depressive Disorder: a voxel-based meta-analysis of functional magnetic resonance imaging studies. Eur. Neuropsychopharmacol. 22, 100-113.

Emsell, L., Langan, C., Van Hecke, W., Barker, G.J., Leemans, A., Sunaert, S., McCarthy, P., Nolan, R., Cannon, D.M., McDonald, C., 2014. White matter differences in euthymic bipolar I disorder: a combined magnetic resonance imaging and diffusion tensor imaging voxel-based study. Bipolar Disorders (in press), electronic publication 29 April, 2013; http://dx.doi.org/10.1111/bdi.12073.

Etkin, A., Egner, T., Peraza, D.M., Kandel, E.R., Hirsch, J., 2006. Resolving emotional conflict: a role for the rostral anterior cingulate cortex in modulating activity in the amygdala. Neuron 51, 871-882.

Favre, S., Aubry, J.-M., Gex-Fabry, M., Ragama-Pardos, E., McQuillan, A., Bertschy, G., 2003. Translation and validation of a French version of the Young Mania Rating Scale (YMRS). Encephale 29, 499-505.

Fernández-Corcuera, P., Salvador, R., Monté, G.C. Salvador Sarró, S., Goikolea, J.M. Amann, B., Moro, N., Sans-Sansa, B., Ortiz-Gil, J., Vieta, E., Maristany, T., McKenna, P.J., Pomarol-Clotet, E., 2013. Bipolar depressed patients show both failure to activate and failure to de-activate during performance of a working memory task. J. Affect. Disord. 148, 170-178.

Foland-Ross, L.C., Bookheimer, S.Y., Lieberman, M.D., Sugar, C.A., Townsend, J.D., Fischer, J., Torrisi, S., Penfold, C., Madsen, S.K., Thompson, P.M., Altshuler, L.L., 2012. Normal amygdala activation but deficient ventrolateral prefrontal activation in adults with bipolar disorder during euthymia. Neuroimage 59, 738-744.

Fox, M.D., Snyder, A.Z., Vincent, J.L., Corbetta, M., Van Essen, D.C., Raichle, M.E., 2005. The human brain is intrinsically organized into dynamic, anticorrelated functional networks. Proc. Natl. Acad. Sci. USA 102, 9673-9678.

Hafeman, D.M., Chang, K.D., Garrett, A.S., Sanders, E.M., Phillips, M.L, 2012. Effects of medication on neuroimaging findings in bipolar disorder: an updated review. Bipolar Disord. 14, 375-410.

Harrison, B.J., Pujol, J., Contreras-Rodríguez, O., Soriano-Mas, C., López-Solà, M., Deus, J., Ortiz, H., Blanco-Hinojo, L., Alonso, P., Hernández-Ribas, R., Cardoner, N., Menchón, J.M., 2011. Task-induced deactivation from rest extends beyond the default mode brain network. PLoS ONE 6, e22964.

Hedborg, K., Muhr, C., 2011. Multimodal behavioral treatment of migraine: an internet-administered, randomized, controlled trial. Upps. J. Med. Sci. 116, 169-186.

Hertel, P.T., 1998. Relation between rumination and impaired memory in dysphoric moods. J. Abnorm. Psychol. 107, 166-172. 
Holtzheimer, P.E., Kelley, M.E. Gross, R.E., Filkowski, M.M., Garlow, S.J., Barrocas, A. Wint, D., Craighead, M.C., Kozarsky, J., Chismar, R., Moreines, J.L., Mewes, K. Posse, P.R., Gutman, D.A., Mayberg, H.S., 2012. Subcallosal cingulate deep brain stimulation for treatment-resistant unipolar and bipolar depression. Arch. Gen. Psychiatry 69, 150-158.

Houenou, J., Frommberger, J., Carde, S., Glasbrenner, M., Diener, C., Leboyer, M., Wessa, M., 2011. Neuroimaging-based markers of bipolar disorder: evidence from two meta-analyses. J. Affect. Disord. 132, 344-355.

Huijbers, W., Pennartz, C.M.A., Cabeza, R., Daselaar, S.M., 2011. The hippocampus is coupled with the default network during memory retrieval but not during memory encoding. PLoS One 6, e17463.

Kaladjian, A., Jeanningros, R., Azorin, J.-M., Nazarian, B., Roth, M., Anton, J.-L., Mazzola-Pomietto, P., 2009. Remission from mania is associated with a decrease in amygdala activation during motor response inhibition. Bipolar Disord. 11, 530-538.

Krebs, R.M., Boehler, CN. De Belder, M., Egner, T, 2013. Neural Conflict-Control Mechanisms Improve Memory for Target Stimuli. Cerebral Cortex (in press), electronic publication 9 October, 2013; http://dx.doi.org/10.1093/cercor/bht283.

Kronhaus, D.M., Lawrence, N.S., Williams, A.M., Frangou, S., Brammer, M.J. Williams, S.C.R., Andrew, C.M., Phillips, M.L., 2006. Stroop performance in bipolar disorder: further evidence for abnormalities in the ventral prefrontal cortex. Bipolar Disord. 8, 28-39.

Lemogne, C., Delaveau, P., Freton, M., Guionnet, S., Fossati, P., 2012. Medial prefrontal cortex and the self in major depression. J. Affect. Disord. 136, e1-e11.

Levens, S.M., Muhtadie, L., Gotlib, I.H., 2009. Rumination and impaired resource allocation in depression. J. Abnorm. Psychol. 118, 757-766.

Malhi, G.S., Lagopoulos, J., Sachdev, P.S., Ivanovski, B., Shnier, R., 2005. An emotional Stroop functional MRI study of euthymic bipolar disorder. Bipolar Disord. 7 (Suppl. 5), S58-S69.

Marchand, W.R., Lee, J.N., Thatcher, J., Thatcher, G.W., Jensen, C., Starr, J., 2007. A preliminary longitudinal fMRI study of frontal-subcortical circuits in bipolar disorder using a paced motor activation paradigm. J. Affect. Disord. 103, 237-241.

Marchetti, I., Koster, E.H.W., Sonuga-Barke, E.J., De Raedt, R., 2012. The default mode network and recurrent depression: a neurobiological model of cognitive risk factors. Neuropsychol. Rev. 22, 229-251.

Mazzola-Pomietto, P., Kaladjian, A., Azorin, J.-M., Anton, J.-L., Jeanningros, R., 2009. Bilateral decrease in ventrolateral prefrontal cortex activation during motor response inhibition in mania. J. Psychiatr. Res. 43, 432-441.

Nee, D.E., Wager, T.D., Jonides, J., 2007. Interference resolution: insights from meta-analysis of neuroimaging tasks. Cognit. Affect. Behav. Neurosci. 7, 1-17.

Ovaysikia, S., Tahir, K.A., Chan, J.L., DeSouza, J.F.X., 2011. Word wins over face: emotional Stroop effect activates the frontal cortical network. Front. Hum. Neurosci. 4, 234.

Pearson, J.M., Heilbronner, S.R., Barack, D.L., Hayden, B.Y., Platt, M.L., 2011. Posterior cingulate cortex: adapting behavior to a changing world. Trends Cognit. Sci. 15 143-151.

Pomarol-Clotet, E., Moro, N., Sarró, S., Goikolea, J.M., Vieta, E., Amann, B. Fernàndez-Corcuera, P., Sans-Sansa, B., Monté, G.C., Capdevila, A., McKenna, P. J., Salvador, R., 2012. Failure of de-activation in the medial frontal cortex in mania: evidence for default mode network dysfunction in the disorder. World J. Biol. Psychiatry 13, 616-626.

Pompei, F., Jogia, J., Tatarelli, R., Girardi, P., Rubia, K., Kumari, V., Frangou, S., 2011 Familial and disease specific abnormalities in the neural correlates of the Stroop Task in Bipolar Disorder. Neuroimage 56, 1677-1684.

Pompei, F., Dima, D., Rubia, K., Kumari, V., Frangou, S., 2011b. Dissociable functional connectivity changes during the Stroop task relating to risk, resilience and disease expression in bipolar disorder. Neuroimage 57, 576-582.
Pourtois, G., Schettino, A., Vuilleumier, P., 2013. Brain mechanisms for emotional influences on perception and attention: what is magic and what is not. Biol. Psychol. 92, 492-512.

Qin, P., Northoff, G., 2011. How is our self related to midline regions and the defaultmode network? Neuroimage 57, 1221-1233.

Roberts, K.L., Hall, D.A., 2008. Examining a supramodal network for conflict processing: a systematic review and novel functional magnetic resonance imaging data for related visual and auditory stroop tasks. J. Cognit. Neurosci. 20, 1063-1078.

Roth, R.M., Koven, N.S., Randolph, J.J., Flashman, L.A., Pixley, H.S., Ricketts, S.M., Wishart, H.A., Saykin, A.J., 2006. Functional magnetic resonance imaging of executive control in bipolar disorder. Neuroreport 17, 1085-1089.

Rottschy, C., Langner, R., Dogan, I., Reetz, K., Laird, A.R., Schulz, J.B., Fox, P.T, Eickhoff, S.B., 2012. Modelling neural correlates of working memory: a coordinate-based meta-analysis. Neuroimage 60, 830-846.

Schwartz, S., Vuilleumier, P., Hutton, C., Maravita, A., Dolan, R.J., Driver, J., 2005 Attentional load and sensory competition in human vision: modulation of fMRI responses by load at fixation during task-irrelevant stimulation in the peripheral visual field. Cereb. Cortex 15, 770-786.

Sheehan, D., Lecrubier, Y., Harnett Sheehan, K., Janavs, J., Weiller, E., Keskiner, A. Schinka, J., Knapp, E., Sheehan, M., Dunbar, G., 1997. The validity of the Mini International Neuropsychiatric Interview (MINI) according to the SCID-P and its reliability. Eur. Psychiatry 12, 232-241.

Strakowski, S.M., Adler, C.M. Almeida, J, Altshuler, L.L., Blumberg H.P., Chang, K.D. DelBello, M.P., Frangou, S., McIntosh, A., Phillips, M.L., Sussman, J.E., Townsend, J.D., 2012. The functional neuroanatomy of bipolar disorder: a consensus model. Bipolar Disord. 14, 313-325.

Strakowski, S.M., Adler, C.M., Holland, S.K., Mills, N., DelBello, M.P., 2004. A preliminary FMRI study of sustained attention in euthymic, unmedicated bipolar disorder. Neuropsychopharmacology 29, 1734-1740.

Strakowski, S.M., Adler, C.M., Holland, S.K., Mills, N.P., DelBello, M.P., Eliassen, J.C. 2005. Abnormal FMRI brain activation in euthymic bipolar disorder patients during a counting Stroop interference task. Am. J. Psychiatry 162, 1697-1705.

Strakowski, S.M., Fleck, D.E., DelBello, M.P., Adler, C.M., Shear, P.K., Kotwal, R., Arndt S., 2010. Impulsivity across the course of bipolar disorder. Bipolar Disord. 12, 285-297.

Svanborg, P., Asberg, M., 1994. A new self-rating scale for depression and anxiety states based on the Comprehensive Psychopathological Rating Scale. Acta Psychiatr. Scand. 89, 21-28.

Townsend, J., Bookheimer, S.Y., Foland-Ross, L.C., Sugar, C.A., Altshuler, L.L., 2010. fMRI abnormalities in dorsolateral prefrontal cortex during a working memory task in manic, euthymic and depressed bipolar subjects. Psychiatry Res.: Neuroimaging 182, 22-29.

Townsend, J., Altshuler, L.L., 2012. Emotion processing and regulation in bipolar disorder: a review. Bipolar Disord. 14, 326-339.

Townsend, J.D., Bookheimer, S.Y., Foland-Ross, L.C., Moody, T.D., Eisenberger, N.I. Fischer, J.S., Cohen, M.S., Sugar, C.A., Altshuler, L.L., 2012. Deficits in inferior frontal cortex activation in euthymic bipolar disorder patients during a response inhibition task. Bipolar Disord. 14, 442-450.

Vederine, F.-E., Wessa, M., Leboyer, M., Houenou, J., 2011. A meta-analysis of wholebrain diffusion tensor imaging studies in bipolar disorder. Prog. Neuropsychopharmacol. Biol. Psychiatry 35, 1820-1826.

Vizueta, N., Rudie, J.D., Townsend, J.D., Torrisi, S., Moody, T.D., Bookheimer, S.Y. Altshuler, L.L., 2012. Regional fMRI hypoactivation and altered functional connectivity during emotion processing in nonmedicated depressed patients with bipolar II disorder. Am. J. Psychiatry 169, 831-840.

Whitfield-Gabrieli, S., Ford, J.M., 2012. Default mode network activity and connectivity in psychopathology. Annu. Rev. Clin. Psychol. 8, 49-76.

Young, R.C., Biggs, J.T., Ziegler, V.E., Meyer, D.A., 1978. A rating scale for mania: reliability, validity and sensitivity. Br. J. Psychiatry 133, 429-435. 